\title{
Systematic design of single-mode coupled-resonator optical waveguides in photonic crystals
}

\author{
Mohammad Soltani and Ali Adibi \\ School of Electrical and Computer Engineering, Georgia Institute of Technology, Atlanta, Georgia 30332-0250 \\ Yong Xu and Reginald K. Lee \\ Department of Applied Physics, 128-95, California Institute of Technology, Pasadena, California 91125
}

Received April 21, 2003

\begin{abstract}
By establishing a direct relation between the dispersion and the field profile of a coupled-resonator optical waveguide (CROW) and those of its constituent cavities, we present a systematic method for the design of a single-mode CROW and for control of its dispersion. The procedure includes the design of a single-mode cavity and control of its frequency by engineering its structure. Then, by chaining these cavities in the proper direction and at an appropriate distance, we achieve the desired dispersion for the CROW. (C) 2003 Optical Society of America

OCIS codes: $130.2790,230.7370,230.5750$.
\end{abstract}

Implementing slow wave structures in microscale devices brings novel applications in optical integrated circuits. Photonic crystals ${ }^{1,2}$ have provided a new means for achieving such structures. These structures exhibit very low group velocity and enable us to design and fabricate new devices for novel applications, including nonlinear optics and ultrashort lasers. ${ }^{3}$

It is known that periodically arranging point defects (or cavities) with weak coupling between them generates a guiding structure called a coupled-resonator optical waveguide (CROW). ${ }^{4,5}$ The dispersion of the structure is narrow in the frequency domain and is in the neighborhood of the resonance frequency of the cavities. Correspondingly, light in a CROW propagates with small group velocity in the defect chain. ${ }^{4-7}$ The coupling strength between the neighboring cavities directly affects the dispersion and the group velocity of the guided modes. Weak coupling results in a narrower dispersion bandwidth and smaller group velocity. One way to decrease the coupling strength is to increase the period of the defect chains ${ }^{4,5,7}$ (i.e., the distance between the adjacent cavities). Another way is to create the defect chain in a particular direction in the photonic crystal such that the coupling strength between the neighboring cavities becomes intrinsically small. ${ }^{, 7}$ Because the guided modes in a CROW result from coupling between individual cavities, the number of guided modes in the CROW depends on the number of cavity modes. For practical applications, single-mode CROWs are preferable.

In this Letter we present a systematic method for the design of a single-mode CROW and maximize the linear dispersion range for the guided mode. The method is based on the engineering of a single cavity and on modifying its structural properties to control its resonance frequencies and field profiles, which are directly related to the properties of the guided modes of the CROW.

Figure 1(a) shows one unit cell of a typical CROW structure in a triangular lattice photonic crystal of air holes in GaAs. We made the defect chain by periodi- cally removing some air holes located in a row along the $\Gamma-M$ direction. Figure $1(\mathrm{~b})$ shows a dispersion diagram of the TM (magnetic field normal to the plane of periodicity) guided modes for the structure shown in Fig. 1(a). For the simulations described in this Letter we used a computer code based on the twodimensional finite-difference time domain ${ }^{8}$ method. The results shown in Fig. 1 were calculated by use of an order- $N$ spectral method. ${ }^{9}$ The computational domain that we used to calculate the dispersion diagrams and field patterns of the guided modes of a CROW is shown in Fig. 1(a). We used Bloch boundary conditions on the right and left sides and perfectly matched layers ${ }^{10}$ on the top and the bottom of the structure. The spectrum and field patterns of the cavity modes were calculated by use of perfectly matched layers on all boundaries. The dielectric material that was used throughout the research reported

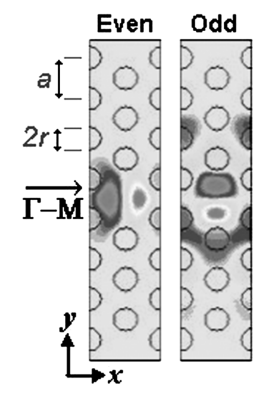

(a)

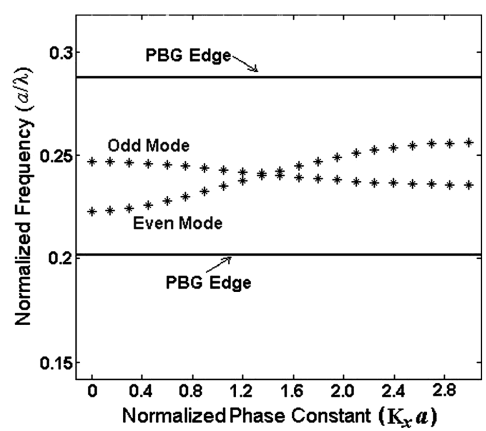

(b)
Fig. 1. (a) One unit cell and the field patterns of the even (left) and odd (right) TM modes of a CROW made by removal of one row of air holes in the $\Gamma-M$ direction of the two-dimensional triangular lattice photonic crystal of air holes in GaAs $(\epsilon=12.96)$. (b) Dispersion diagram of the CROW in the photonic bandgap (PBG). The radius of all air holes is $r=0.3 a$. The direction of propagation is along the $x$ axis, and the magnetic fields for both TM modes are in the $z$ direction (normal to the plane of periodicity). 
in this Letter was GaAs with a relative permittivity of $\epsilon=12.96$, and the material inside the holes was air with $\epsilon=1$. The radius of all the air holes in the triangular-lattice photonic crystal used in this research was $r=0.3 a$ [ $a$ is the lattice constant of the structure as shown in Fig. 1(a)] unless otherwise stated. As shown in Fig. 1(b), the CROW in Fig. 1(a) has two modes. The dispersion diagrams of both the even and the odd modes are nearly flat, yielding a small guiding bandwidth. The dispersion of the odd mode is flatter than that of the even mode, and this fact can be explained by the field profiles of these modes shown in Fig. 1(a). The field profile of the even mode is more horizontally extended, whereas that of the odd mode is more vertically extended. Thus the extension of the even mode at the neighboring cells in the horizontal direction is stronger than that of the odd mode. Therefore the field coupling between adjacent defects is stronger for the even mode than for the odd mode. Stronger field coupling between cavities with the same resonance frequency results in a wider bandwidth of the dispersion diagram of the resultant CROW for the even modes.

For practical applications it is often desirable to achieve single-mode operation and a linear dispersion in a CROW waveguide. But here, as shown in Fig. 1(b), only a small bandwidth lies within the range of single-mode operation, with a highly nonlinear dispersion relation. The existence of more than one guided mode in conventional dielectric-core CROWs has not been extensively investigated in the literature ${ }^{5-7,11}$; only one of the cavity modes has been considered.

The origin of the two guided modes in the CROW in Fig. 1(a) lies in the degeneracy of the cavity modes. Figure 2 shows the field profiles of the even and odd modes and the spectrum of a single cavity used in designing the CROW. The cavity supports two degenerate modes owing to its $C_{6 V}$ symmetry. Coupling these cavities to form a CROW breaks the symmetry, resulting in two modes that cover different frequency ranges. The similarity of the field patterns of the even and odd modes of the cavity to those of the CROW is easily seen from a comparison of Figs. 1 and 2. Furthermore, the frequency extents of the two modes of the CROW are close to the cavity resonance frequency. The actual shapes of the dispersion diagrams of the two modes of the CROW depend on the coupling between the adjacent cavities, with stronger coupling leading to a larger guiding bandwidth. Therefore we can control the number of the guided modes in a CROW and their dispersions by modifying the properties of the corresponding cavity.

The first step in designing a single-mode CROW is to design a cavity with only one mode in the frequency range of interest. We begin with a photonic crystal defect cavity with a single resonance frequency, which can be achieved by proper choice of the hole size and the permittivity contrast of the photonic crystal. Next, we lift the modal degeneracy of the defect cavity by breaking the cavity's $C_{6 V}$ symmetry, which yields two different resonant frequencies for the even and odd modes. The separation of the even and odd bands of the CROW modes is determined to a large degree by the frequency difference of the underlying cavity modes. Besides ensuring single-mode guidance in a CROW, the breaking of the $C_{6 V}$ symmetry also allows us to control the absolute frequency position of the guided modes. More specifically, we can keep the dispersion of the even mode at approximately the same frequency position and push the dispersion of the odd mode outside the gap or separate it completely from the even mode. In the research reported in this Letter we chose to break the cavity symmetry by changing the radii of a pair holes in the defect cavity to $R_{d}>r$, as demonstrated in Fig. 3. The radii of the rest of the air holes are kept at the same value, $r=0.3 a$. The corresponding resonance frequencies of even and odd modes of the cavity for several values of $R_{d}$ are shown in Fig. 3(b).

It is clear from Fig. 3(b) that increasing $R_{d}$ causes the separation between the resonance frequencies of even and odd cavity modes to become larger. Furthermore, it is clear that, whereas the resonance frequency of the even mode remains relatively unaffected by increasing $R_{d}$, that of the odd mode increases almost linearly with increasing $R_{d}$. This result can be intuitively understood from the modal profiles shown in Fig. 3(a). Because of its spatial distribution, the even mode is affected mostly by the two columns of air holes on the right and the left of the cavity. The odd mode, however, is affected mostly by the vertical cavity defined by the two rows of air holes above and below the

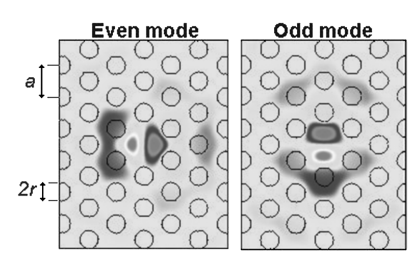

(a)

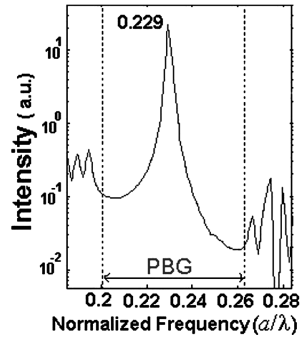

(b)
Fig. 2. (a) Field profiles and (b) spectrum of the TM modes of a single cavity made by removal of one hole from the two-dimensional triangular lattice photonic crystal of air holes in GaAs. The radius of all air holes is $r=0.3 a$. The cavity spectrum is defined as the variation of the electromagnetic field intensity inside the cavity versus frequency.

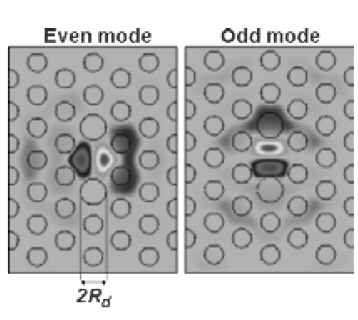

(a)

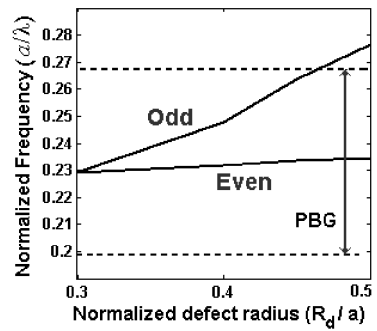

(b)
Fig. 3. (a) Typical field profiles of the modified (nondegenerate) cavity and (b) variation of the resonance frequencies of the TM cavity modes with the radius of modified holes $\left(R_{d}\right)$. The radius of all other holes is $r=0.3 a$. 


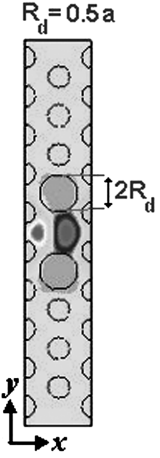

(a)

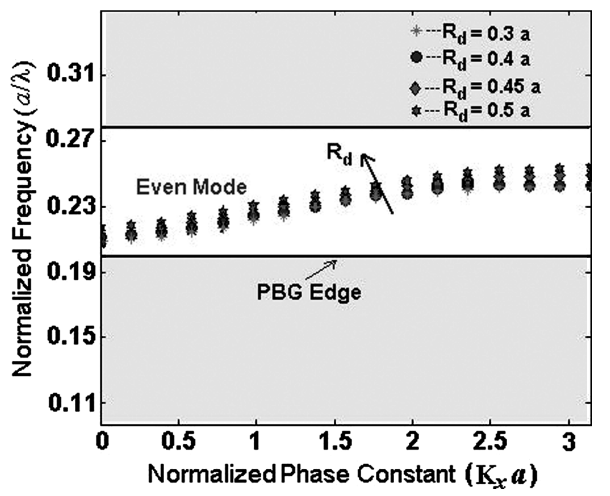

(b)

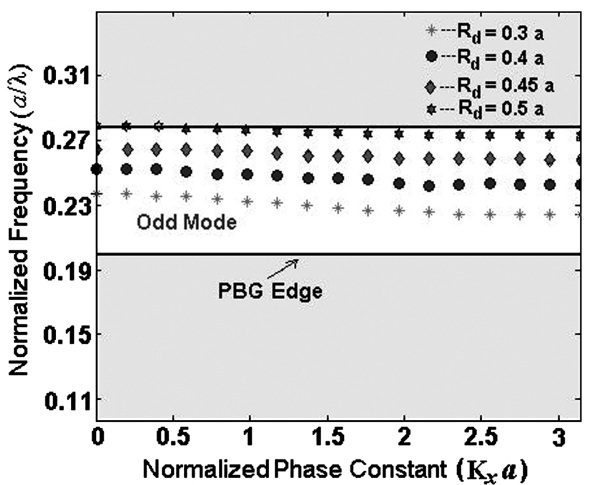

(c)

Fig. 4. (a) One period of the modified CROW and dispersion diagrams of (b) the even and (c) the odd TM modes for different radii of the modified holes $\left(R_{d}\right)$. The field pattern of the even mode at $R_{d}=0.5 a$ is shown in (a). The radius of all other holes is $r=0.3 a$. The direction of propagation is along the $x$ axis, and the magnetic fields for both TM modes are in the $z$ direction (normal to the plane of periodicity).

cavity. Increasing $R_{d}$ does not significantly affect the effective cavity volume for the even mode, but that for the odd mode is reduced, resulting in a higher resonance frequency. One can then construct the modified CROW by chaining these modified cavities.

Figure 4(a) shows one period of such a CROW structure. Figures 4(b) and 4(c) show dispersion diagrams of the even and odd modes, respectively, of the CROW for various sizes of modified holes. As is true for its cavity counterpart, the dispersion of the even mode of the CROW is almost unaffected but that of the odd mode is shifted to higher frequencies and eventually pushed out of the bandgap as $R_{d}$ increases. It can be seen from Fig. 4(b) that for $R_{d}>0.4 a$ the frequency extent of the odd mode has negligible overlap with that of the even mode. Thus the CROW can be used in the normalized frequency range of the even mode as a single-mode waveguide. Furthermore, the CROW is also single mode for the frequency range of the odd mode. Thus we can achieve single-mode guiding over a considerable portion of the photonic bandgap by simply modifying the geometry of a single cavity.

In summary, we have presented a systematic method for designing single-mode CROW in the bandgap of a photonic crystal. The design steps are as follows: 1 , design a single-resonance cavity; 2 , modify the structure of the single cavity to break the degeneracy and separate the resonance frequencies of the degenerate cavity modes, and push the undesired frequency far enough from the desired one; 3, chain the modified cavities with proper periodicity between the adjacent cavities in the direction of propagation. In the end we achieve a single-mode CROW. The guiding bandwidth of the CROW can be controlled and adjusted by proper choices of the period in the guiding direction that controls the coupling between adjacent cavities.

This research was supported by the U.S. Office of Naval Research. A. Adibi's e-mail address is adibi@ece.gatech.edu.

\section{References}

1. E. Yablonovitch, Phys. Rev. Lett. 58, 2059 (1987).

2. S. John, Phys. Rev. Lett. 58, 2486 (1987).

3. M. D. Rahn, A. M. Fox, M. S. Skolnick, and T. F. Krauss, J. Opt. Soc. Am. B 19, 716 (2002).

4. N. Stefanou and A. Modinos, Phys. Rev. B 57, 12,127 (1998).

5. A. Yariv, Y. Xu, R. K. Lee, and A. Scherer, Opt. Lett. 24, 711 (1999).

6. M. Bayindir, B. Temelkuran, and E. Ozbay, Phys. Rev. Lett. 84, 2140 (2000).

7. A. Boag and B. Z. Steinberg, J. Opt. Soc. Am. A 18, 2799 (2001)

8. K. S. Yee, IEEE Trans. Antenna Propag. AP-14, 302 (1966).

9. C. T. Chan, Q. L. Yu, and K. M. Ho, Phys. Rev. B 51, 16,635 (1995).

10. J. P. Berenger, J. Comput. Phys. 114, 185 (1994).

11. A. L. Reynolds, U. Peschel, F. Lederer, P. J. Roberts, T. F. Krauss, and P. J. L. de Maagt, IEEE Trans. Microwave Theory Tech. 49, 1860 (2001). 